\title{
Construction of event-based ISS controllers on coarse quantizations
}

\author{
Lars Grüne and Manuela Sigurani
}

\begin{abstract}
We consider the construction of event-based inputto-state stabilizing state feedback controllers for perturbed nonlinear discrete time systems. The controllers are designed to be constant on possibly coarse quantization regions. An event is triggered upon every transition of the state from one quantization region to another. The practical contribution of the paper is an algorithmic design approach based on game theoretic ideas, feasible for low dimensional systems. The theoretical contribution consists of a novel piecewise constant event-based ISS Lyapunov function concept which is consistent with the imposed quantization.
\end{abstract}

\section{INTRODUCTION}

Event-based control is a useful means to reduce the communication while accomplishing a desired control performance. Besides the investigation of the practical applicability, a lot of effort has been spent on developing a profound theory on event-based control starting with the works of [1], [2] and has been continued in recent years, e.g. by [23], [5], [20], [26], [25]. Most of the literature on event-based control is concerned with stabilization. The problem of rendering the system asymptotically or exponentially stable using eventbased feedback has been studied, among others, by [23], [21], [4], [25], [24]. These event-based control approaches, however, do not tolerate model uncertainties or exogenous disturbances.

In this paper, the event-based structure of the feedback law is induced by an a priori defined, possibly coarse quantization for which an event is generated whenever the state moves from one quantization region to another. Robustness against perturbations and uncertainties is formalized by means of a practical version of input-to-state stability (ISS). The need to consider the practical version of ISS follows immediately from the quantized nature of the controller: since we use only finitely many quantization regions, it is in general only possible to control the state to a neighborhood of a desired equilibrium (in this paper always chosen as the origin).

The dynamic game approach to feedback stabilization, introduced in [8], [9], [12] (extending [18], [7]), was adapted to this setting in [13]. Further results can be found in [10], [6]. In [14], the non-event-based algorithm was utilized to construct an input-to-state practically stabilizing (ISpS) controller for perturbed control systems, using the concept of Lyapunov functions. In this paper we further extend [14] to develop an event-based ISpS controller. An important

This work was supported by the DFG Priority Program 1305, Grant GR1569/11-2 and by the European Union under the 7th Framework Programme FP7-PEOPLE-2010-ITN, Grant agreement nr. 264735-SADCO

L. Grüne and M. Sigurani are with the Mathematical Institute, University of Bayreuth, 95440 Bayreuth, Germany lars.gruene, manuela.sigurani@uni-bayreuth.de auxiliary result proved in this paper is the characterization of the ISpS property for event-based closed loop systems by means of an event-based ISpS Lyapunov function. In all results of this paper we pay particular attention to the influence of the size of the quantization regions on the controller performance. We note that both the Lyapunov function as well as the resulting quantized feedback law are piecewise constant and thus discontinuous in our approach, which is why we provide an analysis entirely avoiding continuity assumptions.

\section{Setting}

Our goal is to construct an event-based input-to-state practically stabilizing (ISpS) controller for the controlled and perturbed discrete-time system

$$
x_{k+1}=f\left(x_{k}, u_{k}, w_{k}\right), \quad k=0,1, \ldots,
$$

using a coarse quantization of the state space $X \subset \mathbb{R}^{d}$. We assume $X$ to be compact and $f(0,0,0)=0$. The discrete time model under consideration can, of course, be the discrete time representation of a sampled continuous time model.

The values $u_{k}$ and $w_{k}$ denote the control and perturbation acting on the system which are taken from sets $U \subset \mathbb{R}^{m}$ and $W \subset \mathbb{R}^{q}$, respectively, which again are supposed to be compact. Infinite sequences of control and perturbation values are denoted by $\mathbf{u}=\left(u_{0}, u_{1}, \ldots\right)$ and $\mathbf{w}=\left(w_{0}, w_{1}, \ldots\right)$ and the corresponding spaces of such sequences with values $u_{k} \in U$ and $w_{k} \in W$ are denoted by $\mathcal{U}$ and $\mathcal{W}$, respectively. The trajectories of (1) are denoted by $x_{k}\left(x_{0}, \mathbf{u}, \mathbf{w}\right)$ or briefly by $x_{k}$ if there is no ambiguity.

We quantize the set $X$ by decomposing it into a finite partition $\mathcal{P}$ of pairwise disjoint regions or cells $P$ with $\bigcup_{P \in \mathcal{P}} P=X$. We let $\rho(x) \in \mathcal{P}, \quad x \in X$, denote the quantization region containing $x$. An event is triggered whenever the trajectory enters a new partition element $P$, i.e., $k \in \mathbb{N}$ is an event time if $\rho\left(x_{k}\right) \neq \rho\left(x_{k-1}\right)$, with the convention that $k=0$ is always an event time. Consequently, a map $u_{\mathcal{P}}: X \rightarrow U$ is an event based controller if it is constant on each region $P \in \mathcal{P}$, i.e., if $u_{\mathcal{P}}\left(x_{k}\right)=u_{\mathcal{P}}\left(x_{k-1}\right)$ whenever $k \in \mathbb{N}$ is not an event time.

The control objective of designing an ISpS controller means that we intend to find an event-based controller $u_{\mathcal{P}}$ such that the closed loop system

$$
x_{k+1}=f\left(x_{k}, u_{\mathcal{P}}\left(x_{k}\right), w_{k}\right), \quad k=0,1, \ldots,
$$

is input-to-state practically stable in the following sense.

Definition 1: System (2) is called input-to-state practically stable (ISpS) with respect to $\delta, \Delta_{w} \in \mathbb{R}_{\geq 0}$ on a set $Y \subset X$ 
if there exist $\beta \in \mathcal{K} \mathcal{L}$ and $\gamma \in \mathcal{K}$, such that the solutions $x_{k}=x_{k}\left(x_{0}, u_{\mathcal{P}}, \mathbf{w}\right)$ of (2) satisfy

$$
\left\|x_{k}\right\| \leq \max \left\{\beta\left(\left\|x_{0}\right\|, k\right), \gamma\left(\|\mathbf{w}\|_{\infty}\right), \delta\right\},
$$

for all $x_{0} \in Y$, all $w \in \mathcal{W}$ with $\|\mathbf{w}\|_{\infty} \leq \Delta_{w}$ and all $k \in \mathbb{N}_{0}$.

The approach we present in this paper relies on the conversion of the ISpS controller design problem into a uniformly practically stabilizing controller design problem. To this end, in the next section we first sketch the dynamic game based design method for uniformly practically stabilizing eventbased controllers from [13]. Afterwards, we explain how to use this approach for the ISpS controller design problem.

\section{GAME THEORETIC STABILIZING CONTROLLER DESIGN FOR PERTURBED SYSTEMS}

In this section we consider the perturbed control system

$$
x_{k+1}=\tilde{f}\left(x_{k}, u_{k}, d_{k}\right), \quad k=0,1, \ldots .
$$

While $w_{k}$ changes to $d_{k}$, state and control $x_{k}$ and $u_{k}$ as well as the respective sets and spaces remain unchanged compared to (1). The precise relation between (1) and (4) will be clarified at the beginning of Section IV. The perturbation values $d_{k}$ are now taken from a set $D \subset \mathbb{R}^{q}$, the corresponding sequences are denoted as $\mathbf{d}=\left(d_{0}, d_{1}, \ldots\right)$ and the space of such sequences with $d_{k} \in D$ is denoted by $\mathcal{D}$. For a given initial state $x \in X$, a given control sequence $\mathbf{u}=\left(u_{k}\right)_{k \in \mathbb{N}} \in \mathcal{U}$ and a given perturbation sequence $\mathbf{d}=\left(d_{k}\right)_{k \in \mathbb{N}} \in \mathcal{D}$, we denote the solution trajectory of (4) by $x_{k}(x, \mathbf{u}, \mathbf{d})$.

The control objective for System (4) is to design a practically uniformly stabilizing event-based controller, i.e., a controller $u_{\mathcal{P}}$ such that the closed loop system

$$
x_{k+1}=\tilde{f}\left(x_{k}, u_{\mathcal{P}}\left(x_{k}\right), d_{k}\right), \quad k=0,1, \ldots .
$$

satisfies Definition 1 with $\gamma=0$.

The relation between the sampling times and the event times is formalized as follows. First, for any $x \in X$, control value $u \in U$ and perturbation sequence $\mathbf{d} \in \mathcal{D}$ we let $j(x, u, \mathbf{d})$ be the time-to-next-event for (4), i.e, the smallest $j \in \mathbb{N}$ with $\rho\left(x_{j}(x, u, \mathbf{d})\right) \neq \rho(x)$. Similarly, we define $j\left(x, u_{\mathcal{P}}, \mathbf{d}\right)$ for (5). Next, for a sequence $\mathbf{x}=\left(x_{k}\right)_{k \in \mathbb{N}_{0}} \in$ $X^{\mathbb{N}_{0}}$ we inductively define the function $\widehat{r}: X^{\mathbb{N}_{0}} \times \mathbb{N}_{0} \rightarrow \mathbb{N}_{0}$ which counts the number of events by $\widehat{r}(\mathbf{x}, 0):=1$ and

$$
\begin{array}{lll}
\widehat{r}(\mathbf{x}, k) & :=\widehat{r}(\mathbf{x}, k-1) & \text { if } \rho\left(x_{k}\right)=\rho\left(x_{k-1}\right) \\
\widehat{r}(\mathbf{x}, k) & :=\widehat{r}(\mathbf{x}, k-1)+1 & \text { if } \rho\left(x_{k}\right) \neq \rho\left(x_{k-1}\right)
\end{array}
$$

for all $k \geq 1$. In order to determine if an event occured, we use the function $\widetilde{r}: X^{\mathbb{N}_{0}} \times \mathbb{N}_{0} \rightarrow\{0,1\}$ given by

$\widetilde{r}(\mathbf{x}, k):=\widehat{r}(\mathbf{x}, k)-\widehat{r}(\mathbf{x}, k-1)= \begin{cases}1 & \text { if an event occured } \\ 0 & \text { if no event occured. }\end{cases}$

Both for theoretical and for computational reasons, we assume that the time-to-next-event $j(x, u, \mathbf{d})$ is bounded by $j(x, u, \mathbf{d}) \leq R$ for an upper bound $R \in \mathbb{N}$. Theoretically, the need for this will become clear in the proof of Case 1 of Theorem 4, below. Computationally, the numerical evaluation of $x_{j(x, u, \mathbf{d})}(x, u, \mathbf{d})$ would take arbitrarily long if $j(x, u, \mathbf{d})$ was unbounded. This upper bound is easily implemented by triggering an event $R$ sampling instants after the last event even if the state did not pass from one quantization region to another. Similarly, we incorporate these artificial events in $\widehat{r}$ and $\widetilde{r}$. We note that this construction is only needed for the design of $u_{\mathcal{P}}$ but not for its implementation.

In order to design a controller we employ the dynamic game approach from [13] which in turn relies on ideas from [8], [9], [18]. To this end we specify a target set $T$ (without loss of generality $T$ assumed to be a quantization region) and a stage cost $g(x, u)$, satisfying the following assumption.

Assumption 2: The stage cost $g$ penalizes the distance to 0 , i.e., there exists $\underline{\alpha} \in \mathcal{K}_{\infty}$ such that

$$
g(x, u) \geq \underline{\alpha}(\|x\|)
$$

holds for all $x \in X, u \in U$.

Then we define the accumulated cost as follows

$$
J(x, \mathbf{u}, \mathbf{d})=\sum_{k=0}^{k(T, x, \mathbf{u}, \mathbf{d})} g\left(x_{k}(x, \mathbf{u}, \mathbf{d}), u_{k}\right),
$$

with $k(T, x, \mathbf{u}, \mathbf{d}):=\inf \left\{k \geq 0 \mid x_{k}(x, \mathbf{u}, \mathbf{d}) \in T\right\}$.

Using the accumulated cost we now define a dynamic game by means of its upper value function

$$
V(x)=\sup _{\beta \in \mathcal{B}} \inf _{\mathbf{u} \in \mathcal{U}} J(x, \mathbf{u}, \beta(\mathbf{u})), \quad x \in X,
$$

where $\mathcal{B}$ denotes the set of all nonanticipating strategies, i.e., all $\beta: \mathcal{U} \rightarrow \mathcal{D}$ satisfying the implication $u_{k}=u_{k}^{\prime} \forall k \leq$ $K \Rightarrow \beta(\mathbf{u})_{k}=\beta\left(\mathbf{u}^{\prime}\right)_{k} \forall k \leq K$ for any $K \in \mathbb{N}_{0}$ and any two control sequences $\mathbf{u}=\left(u_{k}\right)_{k}, \mathbf{u}^{\prime}=\left(u_{k}^{\prime}\right)_{k} \in \mathcal{U}$. By standard dynamic programming arguments [3] one sees that this function fulfills and is uniquely determined by the optimality principle

$$
V(x)=\inf _{u \in U} \sup _{d \in D}\{g(x, u)+V(\tilde{f}(x, u, d))\}
$$

for $x \notin T$ together with the boundary condition $\left.V\right|_{T} \equiv 0$. This equation can also be written as

$$
V(x)=\inf _{u \in U}\left\{g(x, u)+\sup _{x^{\prime} \in \tilde{f}(x, u, D)} V\left(x^{\prime}\right)\right\} .
$$

Note that in (10) the parameterization of $\tilde{f}$ by $d$ is not needed any more since it is sufficient to know the set valued image $\tilde{f}(x, u, D)$.

Next we define the quantized version $V_{\mathcal{P}}$ of the optimal value function $V$. To this end, we first define the event-based cost function on the quantization by

$$
g_{\mathcal{P}}(x, u)=\sup _{x^{\prime} \in \rho(x)} \sup _{\mathbf{d} \in \mathcal{D}} \sum_{k=0}^{j\left(x^{\prime}, u, \mathbf{d}\right)-1} g\left(x_{k}\left(x^{\prime}, u, \mathbf{d}\right), u\right),
$$


and note that Assumption 2 implies $g_{\mathcal{P}}(x, u) \geq \underline{\alpha}(\|x\|)$. We then define the quantized optimal value function by

$$
\begin{aligned}
V_{\mathcal{P}}(x)=\inf _{u \in U} & \left\{g_{\mathcal{P}}(x, u)\right. \\
& \left.+\sup _{x^{\prime} \in \rho(x)} \sup _{\mathbf{d} \in \mathcal{D}} V_{\mathcal{P}}\left(x_{j\left(x^{\prime}, u, \mathbf{d}\right)}\left(x^{\prime}, u, \mathbf{d}\right)\right)\right\}
\end{aligned}
$$

for $x \notin T$ and $V_{\mathcal{P}}(x)=0$ for $x \in T$. Numerically, $V_{\mathcal{P}}$ can be computed by the graph theoretic approach from [9], [13], considering both $d$ and the discretization error as perturbations, cf. [14].

Note that $V_{\mathcal{P}}$ may assume the value $+\infty$ on some parts of $X$, thus we define the stabilizable set w.r.t. $V_{\mathcal{P}}$ by

$$
S_{\mathcal{P}}:=\left\{x \in X \mid V_{\mathcal{P}}(x)<\infty\right\} .
$$

For $x \in S_{\mathcal{P}} \backslash T$, the corresponding feedback $u_{\mathcal{P}}$ is then defined as the minimizer of (12). As desired, it is constant on each partition element $P$.

For $x \in T$ we $\operatorname{set}^{1} u_{\mathcal{P}}(x):=0$ and for $x \in X \backslash S_{\mathcal{P}}$ our approach does not allow for a meaningful definition of $u_{\mathcal{P}}$.

\section{ISPS CONTROLLER DESIGN}

Our controller design approach is based on the first main result in this paper, Theorem 4, below, which characterizes ISpS of an event-based closed loop system by means of an event-based ISpS Lyapunov function $V$. As for its non eventbased counterpart [14], we give a direct proof which allows to determine the resulting gains and the size of the practical stability region. Afterwards we show that $V_{\mathcal{P}}$ from (12) when computed for an appropriate auxiliary system (4) is an ISpS Lyapunov function in this sense for the original closed loop (2). Since an event-based closed loop system is inevitably discontinuous, the classical implication-form ISS Lyapunov function from [15] is not an appropriate concept, cf. [11]. Therefore, we use the strong implication-form ISS-Lyapunov function recently introduced in [11], here adapted to the ISpS property.

Definition 3: A function $V: X \rightarrow \mathbb{R}_{\geq 0}$ which is constant on each quantization region $P \in \mathcal{P}$ is called event-based ISpS Lyapunov function for System (2) on a sublevel set $Y=\{x \in X \mid V(x) \leq \ell\}$ for some $\ell>0$ if there exist functions $\underline{\alpha}, \bar{\alpha} \in \mathcal{K}_{\infty}, \mu, \widetilde{\mu} \in \mathcal{K}$, a positive definite function $\alpha$, values $\bar{w} \in \mathbb{R}_{>0} \cup\{+\infty\}, c, \nu, \widetilde{\nu} \in \mathbb{R}_{\geq 0}$ such that for all $x \in Y$ the inequalities and implications

$$
\underline{\alpha}(\max \{\|x\|-c, 0\}) \leq V(x) \leq \bar{\alpha}(\|x\|)
$$

and

$$
\begin{aligned}
V\left(x_{\tilde{k}}\right)> & \max _{\tilde{k} \leq k<\tilde{\jmath}}\left\{\mu\left(\left\|w_{k}\right\|\right), \nu\right\} \\
& \Rightarrow V\left(x_{\tilde{\jmath}}\right)-V\left(x_{\tilde{k}}\right) \leq-\alpha\left(V\left(x_{\tilde{k}}\right)\right) \\
V\left(x_{\tilde{k}}\right) \leq & \max _{\tilde{k} \leq k<\tilde{\jmath}}\left\{\mu\left(\left\|w_{k}\right\|\right), \nu\right\} \\
& \Rightarrow V\left(x_{\tilde{\jmath}}\right) \leq \max _{\tilde{k} \leq k<\tilde{\jmath}}\left\{\tilde{\mu}\left(\left\|w_{k}\right\|\right), \widetilde{\nu}\right\}
\end{aligned}
$$

${ }^{1}$ If one is willing to use a non-quantized feedback on $T$ then one may also define $u_{\mathcal{P}}$ on $T$ via linearization techniques as, e.g., in [6]. hold for all trajectories $x_{k}$ of (2) in $Y$ corresponding to $w \in$ $W$ with $\|w\| \leq \bar{w}$ and all consecutive event times $\tilde{k}, \tilde{\jmath}$, i.e., all $\tilde{k}, \tilde{\jmath} \in \mathbb{N}$ with $\widetilde{r}(\mathbf{x}, \tilde{k})=\widetilde{r}(\mathbf{x}, \tilde{\jmath})=1$ and $\widehat{r}(\mathbf{x}, \tilde{\jmath})-$ $\widehat{r}(\mathbf{x}, \tilde{k})=1$.

The relation between the existence of an ISpS Lyapunov function and ISpS of the closed loop system (2) is as follows.

Theorem 4: Consider System (2) and assume that the system admits an event-based ISpS Lyapunov function $V$. Then the system is ISpS on $Y=\{x \in X \mid V(x) \leq \ell\}$ with

$$
\begin{aligned}
\delta & =\max \left\{\underline{\alpha}^{-1}(\nu)+c, \underline{\alpha}^{-1}(\widetilde{\nu})+c, 2 c\right\}, \\
\gamma(r) & =\underline{\alpha}^{-1}(\max \{\mu(r), \widetilde{\mu}(r)\})
\end{aligned}
$$

and $\Delta_{w}=\gamma^{-1}\left(\underline{\alpha}^{-1}(\ell)\right)$ for every $\ell>0$ with $\delta \leq \underline{\alpha}^{-1}(\ell)$.

Proof: We fix $x_{0} \in Y$, w $\in \mathcal{W}$ and denote the corresponding trajectory of System (2) with feedback $u_{\mathcal{P}}$ by $x_{k}$. We begin the proof by deriving estimates for $V(x(k))$ under different assumptions. To this end, we denote the event times by $\tilde{k}_{i}, i \in \mathbb{N}$, numbered in ascending order and note that $V\left(x_{\tilde{k}_{i}}\right)=V\left(x_{k}\right)$ for all $k=\tilde{k}_{i-1}, \ldots, \tilde{k}_{i}-1$. Now we distinguish three different cases.

Case 1: Let $i^{\prime} \in \mathbb{N}$ be such that $V\left(x_{\tilde{k}_{i}}\right)>$ $\max \left\{\mu\left(\|\mathbf{w}\|_{\infty}\right), \nu\right\}$ for all $i=0, \ldots, i^{\prime}-1$. Then (15) yields

$$
V\left(x_{\tilde{k}_{i}}\right)-V\left(x_{\tilde{k}_{i-1}}\right) \stackrel{(15)}{\leq}-\alpha\left(V\left(x_{\tilde{k}_{i-1}}\right)\right)
$$

for all $i=1, \ldots, i^{\prime}$. Using a straightforward extension of [17, Lemma 4.3], similar to the proof of Theorem 10 in [14] this implies the existence of $\widehat{\beta} \in \mathcal{K} \mathcal{L}$ such that $V\left(x_{\tilde{k}_{i}}\right) \leq$ $\widehat{\beta}\left(V\left(x_{0}\right), i\right)$ for all $i=0, \ldots, i^{\prime}$. Since $\tilde{k}_{i}-\tilde{k}_{i-1} \leq R$, this implies

$$
V\left(x_{k}\right) \leq \widetilde{\beta}\left(V\left(x_{0}\right), k\right)
$$

for all $k \leq \tilde{k}_{i^{\prime}}$ with $\widetilde{\beta}(r, k)=\widehat{\beta}\left(r,\left\lfloor\frac{k}{R}\right\rfloor\right)+e^{-k} r$, where $\lfloor s\rfloor$ denotes the largest integer $\leq s$. This function $\widetilde{\beta}$ is a $\mathcal{K} \mathcal{L}$ function when restricted to the integers in the second argument. Interpolation yields a $\mathcal{K} \mathcal{L}$ function on $[0, \infty)^{2}$.

Case 2: Let $i \in \mathbb{N}$ be such that $V\left(x_{\tilde{k}_{i-1}}\right) \leq$ $\max \left\{\mu\left(\|\mathbf{w}\|_{\infty}\right), \nu\right\}$. Then (16) yields

$$
V\left(x_{\tilde{k}_{i}}\right) \leq \max \left\{\widetilde{\mu}\left(\|\mathbf{w}\|_{\infty}\right), \widetilde{\nu}\right\} .
$$

Case 3: Let $i \in \mathbb{N}$ be such that $\max \left\{\mu\left(\|\mathbf{w}\|_{\infty}\right), \nu\right\}<$ $V\left(x_{\tilde{k}_{i-1}}\right) \leq \max \left\{\widetilde{\mu}\left(\|\mathbf{w}\|_{\infty}\right), \widetilde{\nu}\right\}$. Then (15) yields

$$
V\left(x_{\tilde{k}_{i}}\right) \leq V\left(x_{\tilde{k}_{i-1}}\right) \leq \max \left\{\widetilde{\mu}\left(\|\mathbf{w}\|_{\infty}\right), \widetilde{\nu}\right\} .
$$

Combining these three cases we can now prove the desired inequality (3):

Let $i^{\prime} \in \mathbb{N}$ be maximal such that the condition from Case 1 is satisfied. Then, for all $k \in\left\{0, \ldots, \tilde{k}_{i^{\prime}}\right\}$ we get

$$
\begin{aligned}
\left\|x_{k}\right\| & \stackrel{(14)}{\leq} \underline{\alpha}^{-1}\left(V\left(x_{k}\right)\right)+c \\
& \stackrel{\alpha^{(18)}}{\leq} \underline{\alpha}^{-1}\left(\widetilde{\beta}\left(V\left(x_{0}\right), k\right)\right)+c \\
& \stackrel{(14)}{\leq} \underline{\alpha}^{-1}\left(\widetilde{\beta}\left(\bar{\alpha}\left(\left\|x_{0}\right\|\right), k\right)\right)+c \\
& \leq \max \left\{2 \underline{\alpha}^{-1}\left(\widetilde{\beta}\left(\bar{\alpha}\left(\left\|x_{0}\right\|\right), k\right)\right), 2 c\right\} .
\end{aligned}
$$

This implies (3) for all $k=0, \ldots, \tilde{k}_{i^{\prime}}$ with $\beta\left(\left\|x_{0}\right\|, k\right):=$ $2 \underline{\alpha}^{-1}\left(\widetilde{\beta}\left(\bar{\alpha}\left(\left\|x_{0}\right\|\right), k\right)\right)$. 
Next, for all $i \geq i^{\prime}$ by induction we show the inequality

$$
V\left(x_{\tilde{k}_{i}}\right) \leq \max \left\{\nu, \widetilde{\nu}, \mu\left(\|\mathbf{w}\|_{\infty}\right), \widetilde{\mu}\left(\|\mathbf{w}\|_{\infty}\right)\right\} .
$$

Note that the bounds on $\delta$ and $\Delta_{w}$ in the assertion ensure that (19) implies $V\left(x_{\tilde{k}_{i}}\right) \leq \ell$ and thus $x_{\tilde{k}_{i}} \in Y$ for all $\mathbf{w} \in \mathcal{W}$ with $\|\mathbf{w}\|_{\infty} \leq \Delta_{w}$. Hence, (19) implies that one of the Cases 1-3 must hold for $x_{\tilde{k}_{i}}$. Consequently, if we know that (19) holds we can use the estimates in the Cases 1-3 in order to conclude an inequality for $V\left(x_{\tilde{k}_{i+1}}\right)$.

To start the induction at $i=i^{\prime}$, note that the maximality of $i^{\prime}$ implies $V\left(x_{\tilde{k}_{i}}\right)<\max \left\{\mu\left(\|\mathbf{w}\|_{\infty}\right), \nu\right\}$ by the condition of Case 1, thus yielding (19).

For the induction step $i \rightarrow i+1$, assume that (19) holds for $x_{\tilde{k}_{i}}$. Then, either Case 1 holds implying $V\left(x_{\tilde{k}_{i+1}}\right) \leq V\left(x_{\tilde{k}_{i}}\right)$ and thus (19) for $V\left(x_{\tilde{k}_{i+1}}\right)$. Otherwise, one of the Cases 2 or 3 must hold for $x_{\tilde{k}_{i}}$ which also implies (19) for $V\left(x_{\tilde{k}_{i+1}}\right)$.

Due to the fact that $V\left(x_{k}\right)$ is constant for $k=$ $\tilde{k}_{i}, \ldots, \tilde{k}_{i+1}-1$, for each $k \geq \tilde{k}_{i^{\prime}}$ (19) together with (14) shows $\left\|x_{k}\right\| \leq \max \left\{\gamma\left(\|\mathbf{w}\|_{\infty}\right), \underline{\alpha}^{-1}(\nu)+c, \underline{\alpha}^{-1}(\widetilde{\nu})+c\right\}$, implying (3) for all $k \geq \tilde{k}_{i^{\prime}}$.

In order to apply the algorithm from the previous section to ISpS controller design we make use of one of the central results in [16], which states that System (2) is ISS if and only if it is robustly stable, i.e., if there exist $e: \mathbb{R}^{n} \times \mathbb{R}^{q} \rightarrow \mathbb{R}^{q}$ and $\eta \in \mathcal{K}_{\infty}$ such that System (5) with $\tilde{f}$ in (4) given by

$$
\tilde{f}(x, u, d)=f(x, u, e(x, d)), \quad D=\bar{B}_{1}(0),
$$

is uniformly asymptotically stable, where $e$ is such that for each $w \in W$ with $\|w\| \leq \eta(\|x\|)$ there exists $d \in D$ with $e(x, d)=w$. For instance, $e$ could be defined as $e(x, d):=$ $\eta(\|x\|) d$ which is also the choice in [16]. The equivalence between ISS and robust stability has been proven for the setting of practical stability in [14] and relies on Lyapunov function arguments.

In the following proposition it is shown that $V_{\mathcal{P}}$ when computed for (20) is an ISpS Lyapunov function for System (2). For its proof we need the following assumption.

Assumption 5: The map $f: X \times U \times D \rightarrow \mathbb{R}^{n}$ in (1) is uniformly continuous in the following sense: there exist $\gamma_{x}, \gamma_{w} \in \mathcal{K}_{\infty}$ such that for all $x, y \in X, u \in U$ and $d \in D$ we have

$\|\tilde{f}(x, u, w)-\tilde{f}(y, u, 0)\| \leq \max \left\{\gamma_{x}(\|x-y\|), \gamma_{w}(\|w\|)\right\}$.

We note that for the closed loop trajectories $x_{k}\left(x, u_{\mathcal{P}}, \mathbf{w}\right)$ of (2), for all $x \in X$ Assumption 5 implies

$$
\begin{array}{r}
\left\|x_{j\left(x, u_{\mathcal{P}}, \mathbf{w}\right)}\left(x, u_{\mathcal{P}}, \mathbf{w}\right)-x_{j\left(x, u_{\mathcal{P}}, \mathbf{0}\right)}\left(x, u_{\mathcal{P}}, \mathbf{0}\right)\right\| \\
\leq \max \left\{\gamma_{w}\left(\left\|w_{j\left(x, u_{\mathcal{P}}, \mathbf{w}\right)-1}\right\|\right), a\right\}
\end{array}
$$

for $a:=\max _{P \in \mathcal{P}, x, y \in P} \gamma_{x}(\|x-y\|)$ and since $u_{\mathcal{P}}(0)=0$ and $f(0,0,0)=0$, for $x \in T$ we get

$$
\begin{aligned}
\left\|x_{j\left(x, u_{\mathcal{P}}, \mathbf{w}\right)}\left(x, u_{\mathcal{P}}, \mathbf{w}\right)\right\| \\
\leq \max \left\{\gamma_{w}\left(\left\|w_{j\left(x, u_{\mathcal{P}}, \mathbf{w}\right)-1}\right\|\right), \theta\right\}
\end{aligned}
$$

for $\theta:=\max _{x \in T} \gamma_{x}(\|x\|)$.

Proposition 6: Consider System (1). Let Assumptions 2 and 5 be satisfied. Let $V_{\mathcal{P}}$ be the quantized optimal value function from (12) constructed for system (20) on a given partition $\mathcal{P}$ with target set $T \in \mathcal{P}$ and $0 \in \operatorname{int} T$. Consider the corresponding feedback $u_{\mathcal{P}}$ defined after (13).

Then $V_{\mathcal{P}}$ is an ISpS Lyapunov function for the closed loop System (2) for any $\ell>0$ with

$$
\begin{aligned}
c & :=\max \{\|x\|: x \in T\} \\
\nu & :=\bar{\alpha}(c) \\
\mu(r) & :=\bar{\alpha}\left(\eta^{-1}(r)\right) \\
\alpha(r) & :=\underline{\alpha}\left(\bar{\alpha}^{-1}(r)\right) \\
\widetilde{\mu}(r) & :=\bar{\alpha}\left(\max \left\{2 \underline{\alpha}^{-1}(\mu(r)), 2 \gamma_{w}(r)\right\}\right) \\
\widetilde{\nu} & :=\bar{\alpha}\left(\max \left\{2 a, \theta, 2 \underline{\alpha}^{-1}(\nu)\right\}\right),
\end{aligned}
$$

where $\underline{\alpha}$ comes from Assumption 2, $\gamma_{w}$ from Assumption 5, $a$ from (21) and $\theta$ from (22) and suitable $\bar{\alpha}$ (see Remark 10, below).

Proof: Proof of (14): If $x \in T$, it follows that $\|x\| \leq c$. Obviously $V_{\mathcal{P}}(x) \geq \inf _{u \in U} g_{\mathcal{P}}(x, u) \geq \inf _{u \in U} g(x, u)$ if $x \notin T$. Due to Assumption 2 we can find an $\underline{\alpha} \in \mathcal{K}_{\infty}$ such that

$$
\begin{aligned}
V_{\mathcal{P}}(x) & \geq \inf _{u \in U} g(x, u) \geq \underline{\alpha}(\|x\|) \quad \forall x \in X \backslash T \\
& \geq \underline{\alpha}(\max \{\|x\|-c, 0\}) \quad \forall x \in X .
\end{aligned}
$$

The existence of an upper bound follows since $V_{\mathcal{P}} \equiv 0$ holds on $T, T$ is a neighborhood of 0 and $V_{\mathcal{P}}$ is piecewise constant and bounded by $\ell$ on $Y$. Hence, $\sup _{x \in Y,\|x\| \leq r} V_{\mathcal{P}}(x)$ is piecewise constant, finite for each $r>0$ and equal to 0 for all sufficiently small $r>0$. Thus, it can be overbounded by a function $\bar{\alpha} \in \mathcal{K}_{\infty}$ which could, e.g., be constructed by piecewise linear interpolation, see also Remark 10.

Proof of (15): Let $\nu:=\bar{\alpha}(c)$. Consider a trajectory $\hat{x}_{k}=$ $\hat{x}_{k}\left(x_{0}, u_{\mathcal{P}}, \mathbf{d}\right)$ of (5) and two consecutive event times $\tilde{k}<\tilde{\jmath}$ with $V\left(\tilde{x}_{\tilde{k}}\right)>\nu$. Then the choice of $\nu$ implies $\hat{x}_{\tilde{k}} \notin T$. Thus,

$$
\begin{aligned}
V_{\mathcal{P}}\left(\hat{x}_{\tilde{\jmath}}\right)-V_{\mathcal{P}}\left(\hat{x}_{\tilde{k}}\right) & \stackrel{(12)}{\leq}-g_{\mathcal{P}}\left(\hat{x}_{\tilde{k}}, u_{\mathcal{P}}\left(\hat{x}_{\tilde{k}}\right)\right) \stackrel{(6)}{\leq}-\underline{\alpha}\left(\left\|\hat{x}_{\tilde{k}}\right\|\right) \\
& \stackrel{(14)}{\leq}-\underline{\alpha}\left(\bar{\alpha}^{-1}\left(V_{\mathcal{P}}\left(\hat{x}_{\tilde{k}}\right)\right)\right)=:-\alpha\left(V_{\mathcal{P}}\left(\hat{x}_{\tilde{k}}\right)\right) .
\end{aligned}
$$

Now consider a trajectory $x_{k}=x_{k}\left(x_{0}, u_{\mathcal{P}}, \mathbf{w}\right)$ of (2). By assumption on $e$ in (20), for all $\mathbf{w} \in \mathcal{W}$ with $\left\|w_{k}\right\| \leq$ $\eta\left(\left\|x_{k}\right\|\right), \quad k \in[\tilde{k}, \tilde{\jmath})$, there exists $\mathbf{d} \in \mathcal{D}$ such that $w_{k}=$ $e\left(x_{k}, d_{k}\right), k \in[\tilde{k}, \tilde{\jmath})$. Since this inequality holds under the condition on the left hand side of $(15)$, for $k=\tilde{k}, \ldots, \tilde{\jmath}$ we can write $x_{k}$ as a trajectory $\hat{x}_{k}$ of (5) and the inequality above implies the right inequality in (15) with $\mu=\bar{\alpha} \circ \eta^{-1}$.

Proof of (16): Let $\tilde{k}<\tilde{\jmath}$ be consecutive event times with $V_{\mathcal{P}}\left(x_{\tilde{k}}\right) \leq \max _{k \in[\tilde{k}, \tilde{\jmath})}\left\{\mu\left(\left\|w_{k}\right\|\right), \nu\right\}$.

If $x_{\tilde{k}} \in T$, then (22) implies

$$
\begin{aligned}
V_{\mathcal{P}}\left(x_{\tilde{\jmath}}\right) & \leq \bar{\alpha}\left(\max \left\{\gamma_{w}\left(\max _{k=\tilde{k}, \ldots, \tilde{\jmath}-1}\left\|w_{k}\right\|\right), \theta\right\}\right) \\
& \leq \max \left\{\tilde{\mu}\left(\max _{k=\tilde{k}, \ldots, \tilde{\jmath}-1}\left\|w_{k}\right\|\right), \widetilde{\nu}\right\} .
\end{aligned}
$$

In case $x_{\tilde{k}} \notin T$, first observe that from the proof of (15) we obtain $\left\|x_{j\left(x_{\tilde{k}}, u_{\mathcal{P}}, \mathbf{0}\right)}\left(x_{\tilde{k}}, u_{\mathcal{P}}, \mathbf{0}\right)\right\| \leq \underline{\alpha}^{-1}\left(V_{\mathcal{P}}\left(x_{\tilde{k}}\right)\right)$. Moreover, 
we have the identity $x_{\tilde{\jmath}}=x_{j\left(x_{\tilde{k}}, u_{\mathcal{P}}, \mathbf{w}_{\tilde{k}+}\right)}\left(x_{\tilde{k}}, u_{\mathcal{P}}, \mathbf{w}_{\tilde{k}+.}\right)$. Together with (21) this implies

$$
\begin{aligned}
& \left\|x_{\tilde{\jmath}}\right\| \leq \| x_{j\left(x_{\tilde{k}}, u_{\mathcal{P}}, \mathbf{w}_{\tilde{k}+}\right)}\left(x_{\tilde{k}}, u_{\mathcal{P}}, \mathbf{w}_{\tilde{k}+.}\right) \\
& -x_{j\left(x_{\tilde{k}}, u_{\mathcal{P}}, \mathbf{0}\right)}\left(x_{\tilde{k}}, u_{\mathcal{P}}, \mathbf{0}\right) \| \\
& +\left\|x_{j\left(x_{\tilde{k}}, u_{\mathcal{P}}, \mathbf{0}\right)}\left(x_{\tilde{k}}, u_{\mathcal{P}}, \mathbf{0}\right)\right\| \\
& \leq \max \left\{\gamma_{w}\left(\left\|w_{j\left(x_{0}, u_{\mathcal{P}}, \mathbf{w}\right)-1}\right\|\right), a\right\} \\
& +\underline{\alpha}^{-1}\left(V_{\mathcal{P}}\left(x_{\tilde{k}}\right)\right) \\
& \leq \max \left\{\gamma_{w}\left(\left\|w_{j\left(x_{0}, u_{\mathcal{P}}, \mathbf{w}\right)-1}\right\|\right), a\right\} \\
& +\underline{\alpha}^{-1}\left(\max _{k \in[\tilde{k}, \tilde{\jmath})}\left\{\mu\left(\left\|w_{k}\right\|\right), \nu\right\}\right) \\
& \leq \max \left\{\max _{k \in[\tilde{k}, \tilde{\jmath})} 2 \gamma_{w}\left(\left\|w_{k}\right\|\right), 2 a,\right. \\
& \left.2 \underline{\alpha}^{-1}\left(\max _{k \in[\tilde{k}, \tilde{\jmath})}\left\{\mu\left(\left\|w_{k}\right\|\right)\right\}\right), 2 \underline{\alpha}^{-1}(\nu)\right\}
\end{aligned}
$$

which again implies

$$
V_{\mathcal{P}}\left(x_{\tilde{\jmath}}\right) \leq \max \left\{\tilde{\mu}\left(\max _{k=\tilde{k}, \ldots, \tilde{\jmath}-1}\left\|w_{k}\right\|\right), \widetilde{\nu}\right\} .
$$

Thus, in both cases we obtain the desired inequality.

Note that since $V_{\mathcal{P}}$ assumes only finitely many different values and is finite on $S_{\mathcal{P}}$, choosing $\ell:=\max _{x \in S_{\mathcal{P}}} V_{\mathcal{P}}(x)$ yields the maximal possible domain $Y=S_{\mathcal{P}}$ on which $V_{\mathcal{P}}$ is an ISpS Lyapunov function.

The second main result of this paper now summarizes the conditions under which the feedback $u_{\mathcal{P}}$ indeed renders System (1) ISpS.

Theorem 7: Consider System (1). Let Assumptions 2 and 5 be satisfied. Let $V_{\mathcal{P}}$ be the quantized optimal value function from (12) constructed for system (20) on a given partition $\mathcal{P}$ with target set $T \in \mathcal{P}$ and $0 \in \operatorname{int} T$. Consider the corresponding feedback $u_{\mathcal{P}}$ defined after (13).

Then, for any $\ell \geq \underline{\alpha}(\delta)$ the system is ISpS on $Y=\{x \in$ $\left.X \mid V_{\mathcal{P}}(x) \leq \ell\right\}$ w.r.t. $\delta=\max \left\{\underline{\alpha}^{-1}(\nu)+c, \underline{\alpha}^{-1}(\widetilde{\nu})+c, 2 c\right\}$ with $\underline{\alpha}, \bar{\alpha}, c, \nu, \widetilde{\nu}$ from Proposition 6 and $\Delta_{w}$ from Theorem 4 .

Proof: By Proposition 6 the function $V_{\mathcal{P}}$ is an ISpS Lyapunov function and Theorem 4 is applicable and yields the ISpS property.

Remark 8: Since the computational part of our approach entirely relies on computing a uniformly practically asymptotically stabilizing feedback law for the scaled system (4) by means of the approach from [13], the extension of this algorithm to implement the computation of feedback laws depending not only on the current but also on past values of the state [9] can be readily applied, allowing a reduction of the number of partition elements representing $u_{\mathcal{P}}$. It has thus been used in the computations of our numerical example in the next section.

Remark 9: The stabilizable Set $S_{\mathcal{P}}$ in (13) can be determined a posteriori. Thus once $V_{\mathcal{P}}$ is computed it can be determined whether the quantization was fine enough in order to yield a desired operating region of the controller.
Remark 10: It follows from the maximization in (12) that a refinement $\mathcal{P}^{\prime}$ of a quantization $\mathcal{P}$ yields a smaller optimal value function $V_{\mathcal{P}^{\prime}} \leq V_{\mathcal{P}}$. Hence, the upper bound $\bar{\alpha}$ decreases, too. Moreover, $\nu$ and $\widetilde{\nu}$ in Proposition 6 also decrease when the target are refined. Thus, both the ISpS gain $\gamma$ and the practical stability parameter $\delta$ decrease when refining $\mathcal{P}$ and $T$. Hence, on finer quantizations the closed loop system should be more robust against perturbations. The numerical example in the next section confirms this very intuitive result.

\section{NUMERICAL EXAMPLE}

In order to illustrate our approach we show numerical results for the thermofluid process from [14]. The state of the process consists of the fill level $x_{1}$ and the temperature $x_{2}$ of a liquid in a tank. The inflow of liquid can be controlled by $u_{1}$ and the liquid in the tank can be cooled using $u_{2}$. The perturbations of the water level $w_{1}$ and the temperature $w_{2}$ model the unknown inflow of liquid from a second tank. For a description of the full model see [19, Appendix]. After some simplifications of the equations, the behavior of the tank system is described by the state-space model

$$
\begin{gathered}
\dot{x}_{1}(t)=\frac{1}{0.065}\left(161 \cdot 10^{-6} u_{1}(t)+129 \cdot 10^{-6} \sqrt{w_{1}(t)+0.34}\right. \\
\left.-270 \cdot 10^{-6} \sqrt{x_{1}(t)}\right) \\
\dot{x}_{2}(t)=\frac{1}{0.065 x_{1}(t)}\left(129 \cdot 10^{-6} \sqrt{w_{1}(t)+0.34}\right. \\
\times\left(w_{2}(t)+300-x_{2}(t)\right) \\
\left.+97 \cdot 10^{-6} u_{2}(t)\left(287-x_{2}(t)\right)\right)
\end{gathered}
$$

with $X=[0.25,0.4] \times[290,320], w_{1} \in$ $[-0.09,0.09], w_{2} \in[-20,20]$ and $u_{i} \in[0,1], i=1,2$. For $u^{\star}=(0.481465,0.48466)^{T}$, the equation exhibits the equilibrium $x^{\star}=(0.32,295)^{T}$. Note that $x^{\star}$ is asymptotically stable, hence the goal of our ISpS controller is not to stabilize the system at $x^{\star}$ but to increase the robustness of the stability against perturbations.

As the system has a cascaded (or triangular) structure i.e., the first equation does not depend on $x_{2}-$ it has turned out beneficial to choose $e$ in (20) to reflect this structure, i.e., to have the first component independent of $x_{2}$ (the proof of Proposition 6 can be adapted to this cascaded situation). Hence we chose $e$ in (20) as

$$
e(x, d)=\left(\begin{array}{c}
\sqrt{1.25297\left(x_{1}-x_{1}^{\star}\right)^{2}} d_{1} \\
\sqrt{618.75\left(x_{1}-x_{1}^{\star}\right)^{2}+0.6273\left(x_{2}-x_{2}^{\star}\right)^{2}} d_{2}
\end{array}\right) .
$$

We computed the controller using the stage cost $g(x, u)=$ $4 \cdot 10^{4}\left(x_{1}-x_{1}^{\star}\right)^{2}+\left(x_{2}-x_{2}^{\star}\right)^{2}$ and the sampling time $2 s$. The control and perturbation value sets $U=[0,1]^{2}$ and $D=[-1,1]^{2}$ were discretized with grids of $9 \times 5$ and $3 \times 3$ equidistant nodes, respectively. In order to show the effect of different quantizations we compare the results for two different partitions $\mathcal{P}$, using $8 \times 8$ equally sized elements 
and $16 \times 16$. In both cases the target $T$ was chosen as the partition element containing $x^{\star}$.

For all trajectory simulations an identical randomly generated sequence $\mathbf{w}$ of perturbations was utilized, using uniformly distributed random numbers in $[0,0.35]$ and $[0,10]$, respectively, for the components of each vector $w_{k} \in \mathbb{R}^{2}$. The resulting trajectories with and without control (for the same sequence w) are shown in Figure 1. One clearly sees that the controllers are able to bring the system considerably closer to the desired equilibrium.

Comparing the controllers calculated with different quantizations, the better disturbance rejection properties of the controller on the finer quantization is clearly visible. The zig-zagging effect of the $x_{1}$-component shows the practical nature of the controller. This effect could be reduced by using a local robust event-based controller near $x^{\star}$ as proposed in [6].
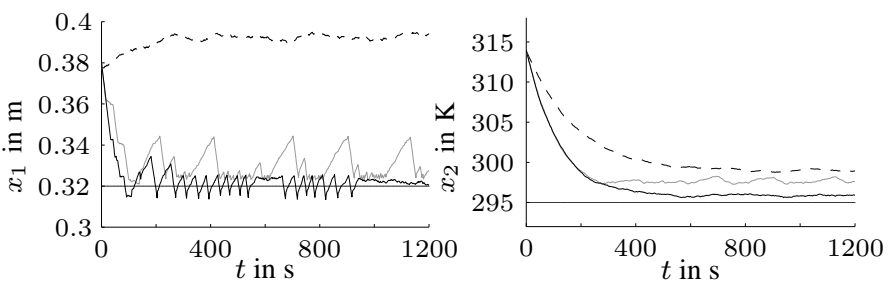

Fig. 1. Trajectories without control (dashed), with control of an $8 \times$ 8 -partition (solid-gray) and of an $16 \times 16$-partition (solid-black), $x_{1}$ component (left) and $x_{2}$-component (right)

\section{CONCLUSION AND OUTLOOK}

We have presented and analyzed a design method for event-based input-to-state stabilizing feedback laws defined on possibly coarse quantizations. The key idea lies in combining a game theoretic approach for event-based stabilization from [13] (relying on graph theoretic algorithms) with a constructive interpretation of the equivalence between ISS and robust stability proved in [16]. The stability proof of the resulting controller relies on a novel sufficient Lyapunov function criterion for input-to-state practical stability in a quantized event based setting. The proofs keep track of all quantitative information like the ISpS gains and the size of the practical stability region.

In future research we intend to use the proposed approach as a building block for a distributed event-based feedback design for large networks of systems, an approach for which first promising experimental results are already available [22]. To this end, an event-based version of the ISS small gain theorem is currently under investigation.

\section{REFERENCES}

[1] K. Arzén, "A simple event-based PID controller," in Proc. 14th IFAC World Congress, 1999, pp. 423-428.
[2] K. J. Åström and B. Bernhardsson, "Comparison of periodic and eventbased sampling for first-order stochastic systems," in Proc. 14th IFAC World Congress, 1999, pp. 301-306.

[3] D. P. Bertsekas, Dynamic Programming and Optimal Control. Vol. 1 and 2. Belmont, MA: Athena Scientific, 1995.

[4] C. De Persis, R. Sailer, and F. Wirth, "On a small-gain approach to distributed event-triggered control," in Proc. 14th IFAC World Congress, 2011, pp. 2401-2406.

[5] P. J. Gawthrop and L. B. Wang, "Event-driven intermittent control," International Journal of Control, vol. 82, pp. 2235-2248, 2009.

[6] L. Grüne, S. Jerg, O. Junge, D. Lehmann, J. Lunze, F. Müller, and M. Post, "Two complementary approaches to event-based control," at-Automatisierungstechnik (Special Issue on Networked Control Systems), vol. 58, pp. 173-182, 2010.

[7] L. Grüne and O. Junge, "A set oriented approach to optimal feedback stabilization," Systems Control Lett., vol. 54, no. 2, pp. 169-180, 2005.

[8] - "Approximately optimal nonlinear stabilization with preservation of the Lyapunov function property," in Proceedings of the 46th IEEE Conference on Decision and Control, New Orleans, Louisiana, 2007, pp. 702-707.

[9] _ _ "Global optimal control of perturbed systems," J. Optim. Theory Appl., vol. 236, no. 3, pp. 411-429, 2008.

[10] - "Set oriented construction of globally optimal controllers," atAutomatisierungstechnik, vol. 57, pp. 287-295, 2009.

[11] L. Grüne and C. Kellet, "ISS-Lyapunov functions for discontinuous discrete-time systems," 2013, preprint, University of Bayreuth, 24 pages. Provisionally accepted in IEEE Trans. Autom. Control.

[12] L. Grüne and F. Müller, "Set oriented optimal control using past information," in Proc. 18th International Symposium on Mathematical Theory of Networks and Systems (MTNS2008), CD-Rom, Paper 125.pdf, Blacksburg, Virginia, 2008.

[13] _ , "An algorithm for event-based optimal feedback control," in Proceedings of the 48th IEEE Conference on Decision and Control, Shanghai, China, 2009, pp. 5311-5316.

[14] L. Grüne and M. Sigurani, "Numerical ISS controller design via a dynamic game approach," in Proceedings of the 52nd IEEE Conference on Decision and Control - CDC 2013, Florence, Italy, 2013, pp. 1732-1737.

[15] Z.-P. Jiang and Y. Wang, "Input-to-state stability for discrete-time nonlinear systems." Automatica, vol. 37, no. 6, pp. 857-869, 2001.

[16] — , "Input-to-state stability for discrete-time nonlinear systems." Automatica, vol. 37, no. 6, pp. 857-869, 2001.

[17] _ "A converse Lyapunov theorem for discrete-time systems with disturbances," Systems Control Lett., vol. 45, no. 1, pp. 49-58, 2002.

[18] O. Junge and H. M. Osinga, "A set oriented approach to global optimal control," ESAIM Control Optim. Calc. Var., vol. 10, no. 2, pp. 259-270 (electronic), 2004.

[19] J. Lunze, Ed., Control Theory of Digitally Networked Systems. Springer, 2014.

[20] J. Lunze and D. Lehmann, "A state-feedback approach to event-based control." Automatica, vol. 46, no. 1, pp. 211-215, 2010.

[21] M. Mazo and P. Tabuada, "Decentralized event-triggered control over wireless sensor/actuator networks," IEEE Transactions on Automatic Control, vol. 56, pp. 2456-2461, 2010.

[22] M. Sigurani, C. Stöcker, L. Grüne, and J. Lunze, "Experimental evaluation of two complementary decentralized event-based control methods," Preprint, University of Bayreuth, 2013, submitted.

[23] P. Tabuada, "Event-triggered real-time scheduling of stabilizing control tasks," IEEE Transactions on Automatic Control, vol. 52, pp. 1680 $1685,2007$.

[24] X. Wang and M. D. Lemmon, "Attentively efficient controllers for event-triggered feedback systems," in Proc. Joint IEEE Conference on Decision and Control and European Control Conference, 2011, pp. 4698-4703.

[25] _ "On event design in event-triggered feedback systems," Automatica, vol. 47, pp. 2319-2322, 2012.

[26] H. Yu and P. J. Antsaklis, "Event-triggered real-time scheduling for stabilization of passive and output feedback passive systems," in Proc. American Control Conference, 2011, pp. 1674-1679. 